\title{
Simplified hemostatic technique during laparoscopic partial nephrectomy
}

\author{
Alexander Tsivian, Matvey Tsivian, Shalva Benjamin, Ami A. Sidi \\ Department of Urologic Surgery, The E. Wolfson Medical Center, Holon and Sackler Faculty of \\ Medicine, Tel Aviv University, Tel Aviv, Israel
}

\section{ABSTRACT}

Introduction: Laparoscopic partial nephrectomy (LPN) has gained popularity in recent years, although it remains a challenging procedure. Herein we describe our technique of renal defect closure using sutures as the sole means of hemostasis during LPN.

Surgical technique: The kidney is approached transperitoneally in a standard fashion. After the renal artery is clamped and the tumor has been excised, the defect is closed in two separate knot-free suture layers. The deep layer suture is continuous and involves deep parenchyma including the collecting system, if opened. The superficial layer suture approximates the margins of the defect using absorbable clips on one parenchymal edge only. No bolsters, glues or other additional hemostatic agents are used.

Results: At present this technique was applied in 34 patients. Tumor size ranged from 17-85 mm. Median warm ischemia time was 23 min (range 12-45) and estimated blood loss $55 \mathrm{~mL}$ (30-1000). There were no intraoperative complications or conversions to open surgery. No urine leaks or postoperative bleedings were observed.

Conclusions: This simplified technique appears reliable and quick, and therefore may be attractive for many urologic surgeons. Furthermore, the avoidance of routine use of additional hemostatic maneuvers may provide an economical advantage to this approach with no compromise of the surgical outcome.

\section{ARTICLE INFO}

\section{Key words:}

laparoscopy; kidney;

nephrectomy; surgery;

hemostatic techniques

Int Braz J Urol. 2012; 38: 84-88

Submitted for publication:

March 03, 2011

Accepted after revision:

August 02, 2011

\section{INTRODUCTION}

Laparoscopic partial nephrectomy (LPN) for the treatment of selected patients with a renal mass has gained popularity over recent years, although it remains a challenging and highly advanced laparoscopic procedure. The most demanding step during LPN is the suturing of the renal defect both because it requires advanced laparoscopic suturing skills and because the suture is performed under time pressure to minimize warm ischemia time. In addition to sutures, hemostatic agents such as glues, bolsters and argon laser, either alone or in combination, are often used during this step to achieve hemostasis (1).

The purpose of this study is to describe a technique of suturing renal defects during LPN that is quick and reliable, does not require additional hemostatic agents, and therefore represents an attractive option for urologic laparoscopic surgeons. 


\section{SURGICAL TECHNIQUE}

The standard transperitoneal approach is used with 3-4 trocars as described elsewhere (2). Briefly, once the tumor has been identified and the incision track marked by cautery, the renal artery is clamped and the tumor excised in a standard fashion using cold scissors and suction. The specimen then is removed from the field.

The closure of the renal defect proceeds in two layers: the first layer comprises the tumor bed and the collected system, if opened. A single running suture is used for this deep layer and secured on both ends by absorbable clips (LapraTy ${ }^{\circledR}$, Ethicon, Cincinatti, $\mathrm{OH}$ ). Ureteral catheters or stents are not used in our institution to verify watertight closure or to protect collecting system closure. The second suture layer comprises the remaining kidney parenchyma. For this layer we use an interrupted running suture as illustrated in Figures $1 \mathrm{~A}$ and $\mathrm{B}$ : the suture thread is prepared with an absorbable clip on its edge. At each passage through the parenchyma, a clip is applied to the thread after approximating the edges, so that the suture is secured with clips on both parenchymal edges upon completion of the first passage (Figure-1C). For subsequent passages, the clips are applied only on one side of the defect (Figures 1D and E). An additional clip may be applied proximally to increase tension. Additional suture passages are carried out as necessary, depending on the size of the renal defect (Figures 1G-F).

No adjunctive hemostatic measures, such as bolsters or glues, are taken, and so the suture is the exclusive means of hemostasis. The renal artery is then unclamped and adequate hemostasis is verified. The remaining of the procedure is carried out in the standard fashion.

The above-described suture technique in LPN was performed on 34 patients over the last 1.5 years (from July 2007 to February 2009). Patient and tumor characteristics are detailed in Table- 1 . We considered hilar tumors as those in direct contact with major renal vessels and/or renal pelvis, and central tumors were defined as masses abutting the collecting system; the remainder of the masses were considered peripheral. The median tumor size was $30 \mathrm{~mm}$ (range 17-85). The surgical
Table 1 - Patient and tumor characteristics.

\begin{tabular}{|c|c|}
\hline Variable & $\begin{array}{c}\text { Median (range)/number } \\
(\%)\end{array}$ \\
\hline Age, years & $62.5(39-78)$ \\
\hline \multicolumn{2}{|l|}{ Gender } \\
\hline Male & $17(50)$ \\
\hline Female & $17(50)$ \\
\hline \multicolumn{2}{|l|}{ Side } \\
\hline Right & $18(53)$ \\
\hline Left & $16(47)$ \\
\hline Tumor size, mm & $30(17-85)$ \\
\hline \multicolumn{2}{|l|}{ Tumor position } \\
\hline Peripheral & $9(26)$ \\
\hline Central & $17(50)$ \\
\hline Hilar & $8(24)$ \\
\hline \multicolumn{2}{|l|}{ Tumor polar location } \\
\hline Upper pole & $13(38)$ \\
\hline Interpolar & $7(21)$ \\
\hline Lower pole & $14(41)$ \\
\hline \multicolumn{2}{|l|}{ Tumor surface location } \\
\hline Anterolateral & $16(47)$ \\
\hline Medial & $3(9)$ \\
\hline Posterior & $15(44)$ \\
\hline Operative time, mins & $150(100-270)$ \\
\hline WIT & $23(12-45)$ \\
\hline EBL, CC & $55(30-1000)$ \\
\hline \multicolumn{2}{|l|}{ Tumor pathology } \\
\hline RCC & $24(70)$ \\
\hline AML & $3(9)$ \\
\hline Oncocytoma & $6(18)$ \\
\hline Cystic nephroma & $1(3)$ \\
\hline
\end{tabular}

WIT: Warm ischemia time

EBL: Estimated blood loss

RCC: Renal cell carcinoma

AML: Angiomyolipoma 
Figure 1 - Sequential steps of renal defect closure during laparoscopic partial nephrectomy with a refined technique

A

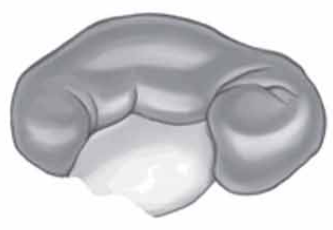

B

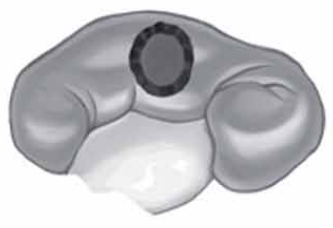

C

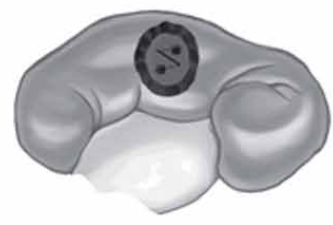

D

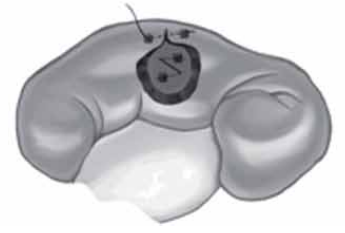

$\mathbf{E}$

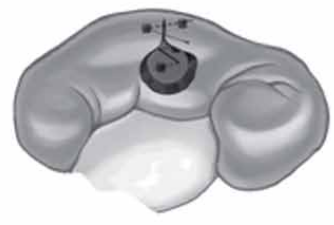

$\mathbf{F}$

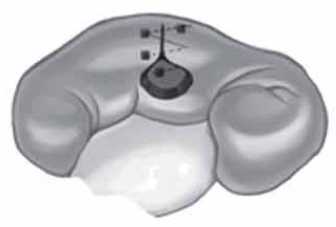

$\mathbf{G}$

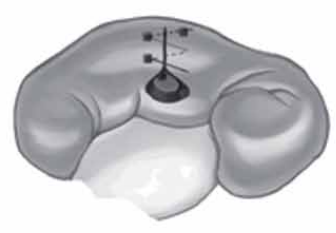

H

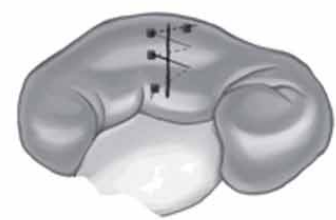

margins were positive in one case. The median warm ischemia time was $23 \mathrm{~min}$ (range 12-45) and estimated blood loss was $55 \mathrm{~mL}$ (range 30-1000). There were no intraoperative complications and none of the 34 procedures was converted to open surgery. No urine leak was observed in the postoperative period. One delayed bleeding occurred 2 weeks after the procedure and was treated angiographically.

\section{COMMENTS}

Renal defect closure in LPN is usually accomplished by additional hemostatic measures (bolsters, glues, argon laser) on top of sutures (1). The simplified method of renal defect closure we devised and now describe does not require the use of additional hemostatic maneuvers other than suturing. This technical modification may reduce warm ischemia time and, therefore, possible damage to the organ. Although no direct comparison is made, the median warm ischemia time in this series was 23 minutes indicating a possible advantage of this hemostatic technique. Warm ischemia time during LPN can be divided into ischemia due to tumor resection and ischemia due to defect closure. The technique we describe affects the latter and, in our experience, is able to sensibly reduce warm ischemia time during this procedure by obviating the need to use bolsters, sealant or argon laser for achieving hemostasis.

Weight et al. (3) were able to avoid bolstered renorrhaphy in select patients using single layered suturing and thrombin sealant. Similarly, Nadu et al. (4) reported using no bolsters in renorrhaphy and closing the defect in two layers and enhancing hemostasis with biological glue. Orvieto et al. (5) used bolstered only in larger defects that could not be approximated by sutures alone, and Agarwal et al. (6) concluded that bolsters were not instrumental for hemostasis. Although a recent review article (7) referred to suturing over 
the bolster as the gold standard, to the best of our knowledge, no study has demonstrated the advantage of the bolster over conventional suturing for renal defect closure. Nevertheless, many laparoscopic surgeons use bolstered renorrhaphy, probably because it "feels" safer.

Synthetic and biological glues for hemostasis were introduced to facilitate hemostasis during LPN and a variety of different compounds is commercially available. However, several authors have reported that they do not use sealants during LPN and have equally satisfactory results $(2,5,6)$. To date, the advantages of such sealants have yet to be clarified. It is our impression that the use of these materials also often falls under the category of the aforementioned surgeon's "feeling" safer.

We did not carry out a cost analysis, but it is reasonable to assume that avoiding routine application of additional hemostatic agents may reduce surgical costs without compromising surgical outcome. In our center, routine use of bolsters and sealants in LPN has been abandoned since the introduction of this new technique.

Standardizing the surgical technique is beneficial not only for the surgeon performing LPN but for those who learn it as well. Closing the defect in 2 layers regardless of whether or not the collecting system is opened represents another advantage of this technique refinement that brings to a further standardization of the surgical technique in LPN that may increase reproducibility and facilitate learning.

Finally, this technique does not preclude the use of additional hemostatic maneuvers if necessary. Although additional hemostasis maneuvers may be required in some cases, their routine use, in our experience, does not seem to provide substantial benefits. It seems to us that whenever the wound requires suturing, the latter may be relied upon as the only hemostatic measure. The advantages and disadvantages of each surgical technique are clearly difficult to compare, but large randomized studies may help provide hard evidence in this surgeon-depending setting.

We no longer routinely use additional hemostatic methods during LPN when the renal defect is sutured. During this study period, 46 patients underwent LPN in our institution and in 34 cases suturing was the only hemostatic maneuver, whereas the remaining 12 cases either pertain to the initial experience when this technique was developing or did not require suturing (superficial tumors). We did not directly compare cases with and without additional hemostatic modalities because of inherent multiple biases that would have affected the applicability of the findings. This study demonstrates the feasibility of renorrhaphy without additional hemostatic maneuvers and suggests that the routine use of bolsters, glues and/or argon laser should be reconsidered.

Our technique obviates the use of bolsters and glues for hemostasis during LPN and may represent an attractive option for many urologists for several reasons. First, this technique is simple and applicable whether or not the collecting system is opened. Second, with this technique it is reasonable to expect reduced costs since no hemostatic bolsters or glues are used. In our opinion, additional hemostatic agents such as bolsters and sealants should be reserved for selected cases and not be used routinely.

\section{CONFLICT OF INTEREST}

None declared.

\section{REFERENCES}

1. Msezane LP, Katz MH, Gofrit ON, Shalhav AL, Zorn KC: Hemostatic agents and instruments in laparoscopic renal surgery. J Endourol. 2008; 22: 403-8.

2. Tsivian A, Shtricker A, Benjamin S, Sidi AA: Laparoscopic partial nephrectomy for tumor excision in a horseshoe kidney. Eur Urol. 2007; 51: 1132-3.

3. Weight CJ, Lane BR, Gill IS: Laparoscopic partial nephrectomy for selected central tumors: omitting the bolster. BJU Int. 2007; 100: 375-8.

4. Nadu A, Mor Y, Laufer M, Winkler H, Kleinmann N, Kitrey $\mathrm{N}$, et al.: Laparoscopic partial nephrectomy: single center experience with 140 patients--evolution of the surgical technique and its impact on patient outcomes. J Urol. 2007; 178: 435-9; discussion 438-9.

5. Orvieto MA, Chien GW, Tolhurst SR, Rapp DE, Steinberg GD, Mikhail AA, et al.: Simplifying laparoscopic partial nephrectomy: technical considerations for reproducible outcomes. Urology. 2005; 66: 976-80. 
6. Agarwal D, O'Malley P, Clarke D, Rao R: Modified technique of renal defect closure following laparoscopic partial nephrectomy. BJU Int. 2007; 100: 967-70.
7. van Dijk JH, Pes PL: Haemostasis in laparoscopic partial nephrectomy: current status. Minim Invasive Ther Allied Technol. 2007; 16: 31-44.

Correspondence address:

Dr. Tsivian Alexander

Dept. of Urologic Surgery the E. Wolfson Medical Center

P.0.Box 5, Holon, 58100, Israel

Fax: + 9723 502-8199

E-mail: atsivian@hotmail.com 